\title{
M3G: A Mobile Multicast Multimedia Gateway for Seamless IPv4/IPv6 Transition
}

\author{
Yassine Hadjadj Aoul, Daniel Negru, Abdelhamid Nafaa, \\ and Ahmed Mehaoua \\ CNRS-PRiSM Lab., University of Versailles \\ 45, av. des Etats Unis 78035 - Versailles - France \\ Tel: +33139254056 \\ Fax: +33139254057 \\ \{yana, dan, anaf, mea\}@prism.uvsq.fr
}

\begin{abstract}
A growing interest in third generation wireless IP network and service technologies, push up the demand on IPv6 transition. Most of these services require mobility, multicast and multimedia service supports. However, the transition to such a NG IP networks and services is slowed down because of the richness and complexity of the applications and the integration of legacy systems. Existing IETF and academic proposals permit to solve several transition problems but they don't cope with the multimedia or wireless aspects of some next generation IP services. In the other hand, most transition solutions are limited to LANs and couldn't scale well. Therefore, this article describes the architecture and the implementation of an integrated multi-functional IP gateway for IPv4/IPv6 network transition with multimedia, multicast and mobility service management. This gateway, named M3G, has been partially implemented and validated on a Linux-based testbed with MPEG4 video streaming applications. Nevertheless, the main goal of this work is to achieve a transparent establishment and a smooth control of unicast (e.g. VOD) and multicast (e.g. TV broadcasting) multimedia communications between heterogeneous fixed and mobile hosts belonging to dense IPv4 and IPv6 domains.
\end{abstract}

\section{Introduction}

The transition from the former version of IP to its new shape is certainly not the least task to consider. Even though IPv6 has a strong base and is easily extensible, the migration is facing difficulties. Therefore, there is a critical need to deploy a solution that would allow IPv4 existing services to be accessed by IPv6 terminals.

The solution proposed in here takes into consideration this aspect. Not only is the heterogeneous problem taken into account at all levels, but also many important features of IPv6 are exploited, from mobility to multicast, including multimedia and scalability. The fruition would be a Mobile Multicast IPv4/IPv6 Gateway for Multimedia Applications working in a heterogeneous environment. Its main role is to manage connectivity between a native IPv4 domain and a native IPv6 one, all in respect to multimedia applications constraints. 
Existing proposals address the unicast case. In addition, MTP [6] provides an multicast IP translation but present several limitations. Ours provides an IETF fully compliant solution through (1) an IPv4/IPv6 addressing mapping, (2) an RTSP protocol for real-time streaming, (3) a SAP/SDP [2][3][4] protocols for transparent media access, (4) a scalable multicast sessions managements as well as (5) a support for mobility.

First, we will present the context and motivations. Next, we will focus on our proposal and especially on the multicast transition, multimedia, mobility and scalability parts. Then, we will develop the structural aspect of our gateway, from the architectural to the implementation point of view. Finally, we include a short analysis of our gateway's performances.

\section{Context and Motivations}

There is a growing demand on multicast media services due to the will of bandwidth saving. This particularity is very appreciable, especially in the wireless networks. Those will need IPv6 technologies for many reasons the most important being the lack of addresses. Also, most of applications communicate only with IPv4 hosts and can't communicate with IPv6 hosts. So, there is a critical need to deploy a transitory solution in order to allow an IPv4 existing service to be accessed by new IPv6 terminals.

\subsection{Transition to IPv6 and Multimedia Context}

[11] [12] [13] IPv6 is an important part of the future infrastructure of the Internet, as it offers improvements over IPv4. The current shortage of IPv4 addresses presents a large problem for the Wireless industry, which has recently offered Wireless Internet access requiring new demands for IP addresses. Therefore, IPv6 has become the target protocol for the forecoming wireless networks and infrastructures.

The key transition objective is to allow IPv6 and IPv4 hosts to interoperate [14]. A second objective is to allow IPv6 hosts and routers to be deployed in the Internet in a highly diffused and incremental fashion, with few interdependencies. A third objective is that the transition should be as easy as possible for end-users, system administrators, and network operators to understand and carry out.

Concerning multimedia applications, for now, the multicast solution has not been fully adopted in an IPv4 context at a network layer level. On the contrary, IPv6 has been designed with the support of multicast.

The deployment of coherent multimedia multicasting services over IP networks involves frequently some out of band signaling protocols behind the RTP [10] media streams. These protocols (e.g. SAP/SDP, RTSP and others) provide essentially an abstraction to initiation stage and transparent access to media through negotiation, configuration and synchronization. 


\subsection{Mobile IPv6 and Multicast Issues}

Mobility is an integrated part of IPv6; it has been designed to make full support for wireless mobility. The main problem, though, is in the combination of multicast and mobility at a terminal level first, but also at a network one afterwards, for multimedia applications, all this according to QoS characteristics and security constraints.

Providing these services are difficult due to (1) frequent changes of mobile host location and group membership and (2) the stringent QoS requirements of multimedia applications. If a conventional multicast routing protocol is used in wireless mobile networks, several problems may be experienced since existing multicast routing protocols assume static hosts when they construct the multicast delivery tree. To overcome the difficulties, several multicast routing protocols for mobile hosts have been proposed. Although the protocols solve several problems inherent to multicast routing proposals for static hosts, they still have problems such as non-optimal delivery path, tunnel convergence problem, datagram duplication, overheads resulting from frequent reconstruction of a multicast tree, scoping problem, etc....

The current IETF mobile-IP specification proposes two approaches for supporting multicast service to mobile hosts [10]: foreign agent-based multicast (referred to as remote-subscription) and home agent-based multicast (referred to as bi-directional tunneling) [17].

In foreign agent-based multicast, a mobile host has to subscribe to multicast groups whenever it moves to a foreign network. It is very simple scheme and does not require any encapsulations. This scheme has the advantages of offering an optimal routing path and non-existence of duplicate copies of datagrams. However, when mobile host is highly mobile, its multicast service may be very expensive because of the difficulty in managing the multicast tree. Furthermore, the extra delay incurred from rebuilding a multicast tree can create the possibility of a disruption in multicast data delivery.

In home agent-based multicast, data delivery is achieved by unicast mobile IP tunneling via home agent. When a home agent receives a multicast datagram destined for a mobile host, it encapsulates the datagram twice (with the mobile host address and the care-of address of the mobile host) and then transmits the datagram to the mobile host as a unicast datagram. This scheme takes advantage of its interoperability with existing networks and its transparency to foreign networks that a mobile host visits. However, the multiple encapsulation increases the packet size, and the datagram delivery path is non-optimal since each delivery route must pass through a home agent. Furthermore, if multiple mobile hosts that belong to the same home network visit the same foreign network, duplicate copies of multicast datagrams will arrive at the foreign network.

\section{M3G: Proposal of a Mobile Multicast Media Gateway for IPv4/IPv6 Transition}

Our proposal of a Mobile Multicast Media Gateway is based on four principal objectives. First, enabling the transition between an IPv4 domain and an IPv6 one, as well from the unicast as from the multicast point of view. Next, accessing multimedia applications with the support of multicast and signaling processes. Then, presenting 
the mobility aspects and the interactions with multicasting and multimedia constraints. Finally, developing the important issue of scalability, especially for multicast session management.

\subsection{IPv4/IPv6 Translation}

The first objective of the $\mathrm{M} 3 \mathrm{G}$ is to enable IPv4/IPv6 transition between heterogeneous networks, and, above all, to support multicast streams. This task is realized through different points. As a first step, an addressing mapping is obligatory. Afterwards, a solution for multicast signaling translation is proposed in order to avoid the manual setup of the gateway present in the MTP [6] proposal.

Address mapping. The gateway consists of a physical entity connected to two different subnets and implementing a double stack IPv4/IPv6. Its main role is to translate and route packets between a native IPv4 domain and a native IPv6 one.

Our translation is based on the NAT-PT [5] proposal for unicast communications. Unlike SIIT [1], which particularity is to use an "IPv4 mapped address", the unicast mapping used by our NAT-PT solution adds a 96-bits prefix for all communications before the unicast IPv4 address. Owing to this prefix, the packets will be routed through the M3G gateway. This implies the recalculation of the checksum.

The classical mapping technique seen above can not be applied to multicast addresses. So we use a dedicated one to that case, based on the proposition of MTP [6] and named "IPv4-compatible" IPv6 multicast group address.

IGMP/MLD signaling. In order to join IPv4 group by IPv6 hosts, we need interaction between the two domains. The extension is done through a simple mechanism that won't drop IGMP [8] packets sent with a TTL=1 but will translate them to MLD [9] packets also with a TTL=1. The translation is based on the shape of the packet since IGMP and MLD have the same functionalities.

Translating IGMPv2 packet to MLD. IGMPv2 fields are translated to MLD fields as follows:

- Type: Takes the value of the IGMP Type.

- Code: This field doesn't exist in IGMP packet. However, it must be initialized to zero by the sender.

- Checksum: Must be recalculated by the gateway.

Maximum Response Delay: Takes the value of the IGMP Maximum Response Time.

- Reserved: This field doesn't exist in IGMP header. It's a reserved field and must be initialized to zero by the sender.

- Multicast Address: The value of this field represents the "IPv4-compatible" IPv6 multicast group address of the IGMP Group Address field.

Translating MLD packet to IGMPv2. MLD fields are translated to IGMPv2 fields as follows:

- Type: Takes the value of the MLD Type. 
- Maximum Response Time: Takes the value of the Maximum Response Delay MLD field if it is lower than 0xff, it is set to 0xff otherwise.

- Checksum: Must be recalculated by the gateway.

- Group address: Is extracted from the IPv6 address if it's a "IPv4-compatible" IPv6 multicast group address of the MLD Multicast Address field, we must have a pool of IPv4 multicast addresses in our gateway otherwise.

The other MLD fields are ignored by the gateway.

We explain below in a more detailed way our solution for the communication from IPv4 multicast media server to IPv6-only mobile hosts. (See section (4.2): Implementation issues).

\subsection{Multimedia Support}

Industrials actors are interested in multimedia support for their products. However, the classical approaches consider principally the transition problems and avoid multimedia aspects. In this way, we integrate the multimedia dimension in our transition proposal, which treats principally the constraints linked to multicast multimedia applications. So, we first discuss in this section about multimedia services access problems and next, about the efficient multimedia support.

Media Services Access. The primary objective of our gateway design, once an environment for multimedia applications is set up, is to propose solutions for the deployment of heterogeneous multimedia applications, going from real-time to streaming, videoconferencing, video surveillance, radio broadcasting, etc... all of these according to applications exigencies and QoS requirements.

In order to allow a transparent access to multimedia streams, M3G supports the commonly used signaling protocols (e.g. RTSP, SAP, and SDP). Moreover, the M3G architecture is enough flexible to enable other application-level protocols intended to support incoming multimedia services. It should be noted that the majority of multimedia applications induce the use of signaling protocols for session initiation and description. Basically, the signaling protocols provide an abstraction to session parameters and automate the access to multicast groups, especially for SAP/SDP.

Currently, M3G supports SAP/SDP and RTSP protocols for both multicast and unicast multimedia streaming scenarios. The translation of their associated packets is achieved through the tracking of their standardized port numbers at the Translator module (see Fig. 3).

Efficient Multimedia Support. For a more efficient support of multicast multimedia sessions and for scalabilities reasons (see section 4.4), we add in M3G the support for MLD and IGMP protocol. This will permit routers to discover the presence of multicast listeners and then to send packets only if there is at least one listener. Thus, M3G does the translation only if it's necessary. Thereby, the active multimedia sessions won't be slowed down by useless packets.

Also, we pass through heterogeneous domains' limitations by creating an interaction between MLD and IGMP protocols (see sections 3.1 and 4.2 for more 
details). Hence, we permit the creation of multicast multimedia sessions between heterogeneous domains.

\subsection{Mobility Support}

The M3G has been developed with mobility support in mind. One of the primary objectives was to integrate wireless applications, starting at a LAN level and then extending to subnets. No major difficulties are encountered on the wireless LAN part, though. The expansion to several subnets and the apparition of mobility aspects, such as a mobile node willing to receive multicast sessions' flows while moving from a place to another, represents one of the most innovative aspects, especially on the convergence of mobility and multicast.

Mobility Purposes. The increasing demand for mobility in the Internet has created the need for a routing protocol that allows a host to roam in the network. Mobile IP is a solution that enables an IP-host to leave its home link while transparently maintaining all of its present connections and remaining reachable to the rest of the Internet. Mobile IPv4 has been standardized by the IETF and Mobile IPv6 is currently an Internet draft.

An IP address identifies the link on which the host resides. If a host moves to a different link without changing its IP address, there is no information in its IP address about the new point of attachment. Existing routing protocols are therefore not able to deliver datagrams to the mobile host correctly, but always route them to its home link. The purpose of Mobile IPv6 [15] is to enable a mobile host to change its point of attachment to the Internet while still maintaining transport-layer connectivity.

The difficult task is to choose an approach for providing multicast traffic to mobile hosts that respects most multimedia applications' constraints.

Interoperation of Multicast and Mobility. The problem considered here is about providing IP multicast to mobile hosts using Mobile IPv6. We do not forget that the source of a multicast traffic comes from an IPv4 server before crossing the gateway and becoming an IPv6 flow. We also suppose that the sender is a fixed node. Thus, no mobility is involved at the source. It means that, for the moment, the focus is done at the receiver side.

Two approaches can be brought here for the support of multicast by mobile nodes as described in section (2.2).

Multicast group registration on home link: a mobile node registers a multicast group on its home link via its home agent. A tunnel between the MN and the HA is set up.

Multicast group registration on foreign link: a mobile node registers a multicast group on its foreign link, via its local multicast router. Its care-of address is used as the source address.

Each of these two approaches gathers advantages and drawbacks. Our solution conveys both, trying to propose an optimized way to deal with multicast flows to mobile hosts. The main requirement for multimedia applications is the delay. We cannot tolerate a long join delay, therefore the multicast group registration should be done on home link through the HA. For that, we propose an extension of the Binding 
Update: Multicast Registration BU. In order to optimize this solution and not sending all the work to the HA, we only adopt this solution until the Mobile Node is able to register to its border router for getting the multicast flow through this way. At that time, the MN will inform its HA that it doesn't need to receive multicast packets from it, thanks to a Multicast Deregistration BU. From then, the HA will not take care of multicast packets destined to this MN. They will be received from the nearest designed router on its care-of address.

The main drawback of this solution is the increase of the signaling part, since new BUs and exchange messages are used between the entities.

However, a good point is that security can be involved through this method. First, the MN-HA tunnel provides it and then it is possible to use a pseudo return routability procedure between the $\mathrm{MN}$ and the designed router as done for routing optimization in Mobile IPv6 between the CN and the MN. Further work and a deeper look need to be added in this area to fulfill these achievements.

\subsection{Scalability Issues}

Scalability is an important issue for multicast session management; it has not been forgotten in our solution.

Unlike MTP [6], which handles connections and creation of multicast group locally, M3G doesn't manage any connections. It takes principally care of routing of packets after their translations.

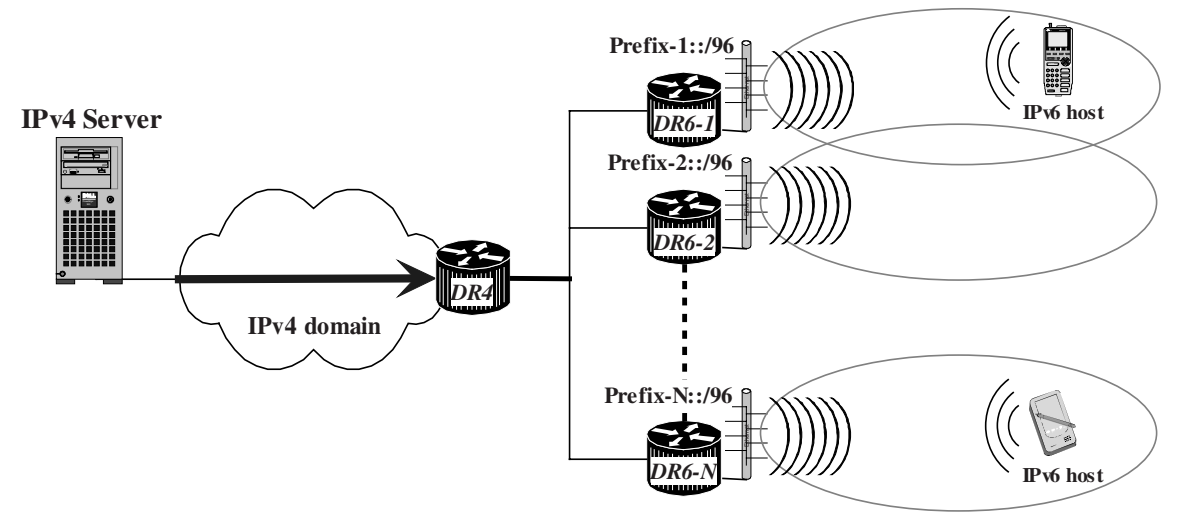

Fig. 1. M3G can be extended almost infinitely by adding M3G boxes. Each M3G box must have an unique prefix (PREFIX::/96) for routing reasons.

Also, we keep DNS information inside the gateway because the DNS has a stateless nature. Thereby, we can limit the use of the gateway and throwback potential bottleneck [7]. This limitation can also be done, by tracking IPv4 SAP/SDP announces in order to avoid redundant costly translations made at application level. Thus, we have to keep the first SAP packet of a given session, after translation, in order to do this translation only once and then send this packet automatically, after the identification of the SAP-4 session. 
An optimization is also done by interacting with IGMP/MLD protocols to translate multicast packets only when there is at least one host wanting to join the multicast group.

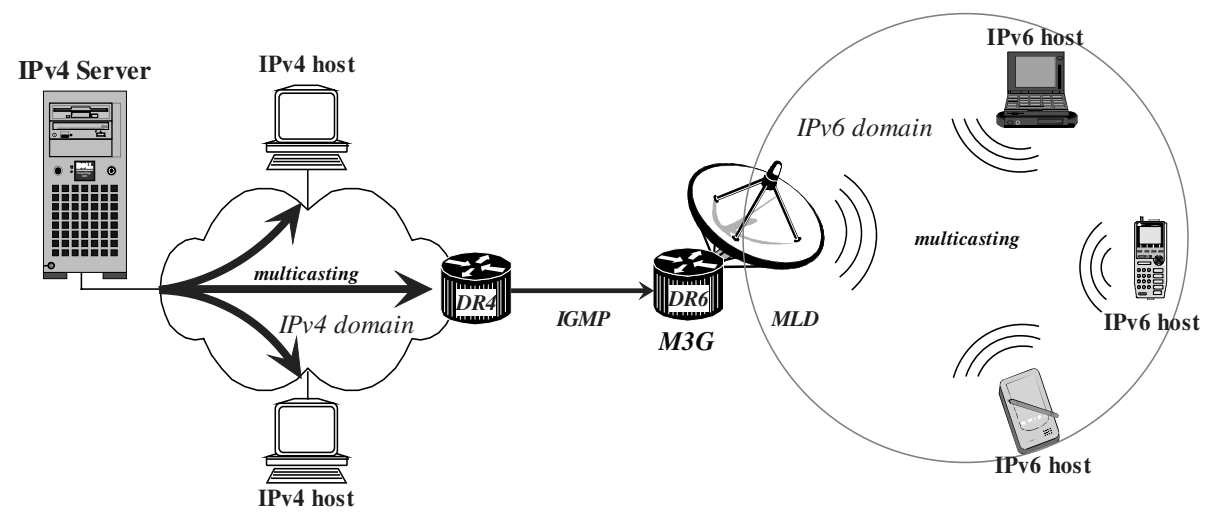

Fig. 2. We consider an IPv6 LAN where some IPv6-only mobile hosts communicate with remaining IPv4 Server in IPv4 domain. We solve the case where IPv6 equipments must talk with IPv4-only domains and vice versa in unicast and multicast. We suppose that no change can be made to the IPv4-only equipments and applications.

\section{Gateway's Structure}

\subsection{M3G Architecture}

The communication is performed through our gateway (M3G) for translating IPv4 packets to IPv6 in order to allow a transparent media access to IPv4 multimedia services. We assume that the router behind our gateway, in the IPv4 domain, implements IGMP protocol. As a result, our gateway will solicit a given multicast stream based only on the clients' requests. According to this fact, M3G considers only the active multicast stream and thus, improves the gateway scalability.

\subsection{Implementation Issues}

\section{Components Specifications}

Link Manager. It operates at a MAC level for the transmission of IPv6 packets, according to RFC 2464 [18] and does the filtering with the use of Berkeley Packet Filters (BPF), thanks to which very low-level programming and interaction with the kernel is done.

Network Manager. It initializes the network functionality and handle IP packets to network modules. 


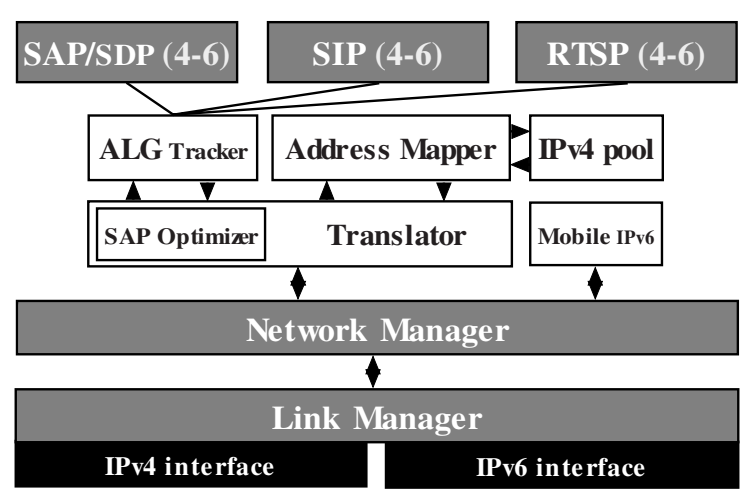

Fig. 3. This section describes in a detailed way the components needed for the whole mechanism and provides an example of the global functioning of the gateway.

Translator. This is the component which translates IPv4 packets into IPv6 ones and vice versa including ICMP and IGMP/MLD packets. The translator includes a "SAP Optimizer" module that permits optimization of communication by sending automatically SAP packets (which have been previously translated).

Mobile IPv6. This module is located at the same level as the translator since Mobile IPv6's signaling process consists of IP level packets. It directly interacts with the translator module by considering IPv6 translated stream. However, it is a complete separate module. It will have to be implemented in the nodes too, either as Home Agent or Mobile Node, depending on what it is desired. It is also possible to set the gateway as a Home Agent.

ALG Tracker. It automatically detects packets concerned with a translation at an application level. Every packet is oriented towards the appropriate module. It will determine by looking into the address field and the port field, if necessary, of translated packets. Therefore, multicast streams will not be translated to the application level and the rapidity of the functioning will increase. Only signaling packets will be translated.

Address Mapper. It maintains each unicast and multicast address pool for IPv4 and IPv6. It also maintains a mapping table which consists of pairs of an IPv4 and an IPv6 address [6]. The translator translates packets between IPv4 and IPv6 domains according to this table. This module is directly connected to the translator, which chooses from it necessary addresses.

SAP/SDP (4-6), SIP (4-6) and RTSP (4-6). These modules do the conversion of packets at the application level. This architecture permits the addition of new modules easily. These signaling packets that need to be converted at the application level are then retransmitted to the desired domain. 


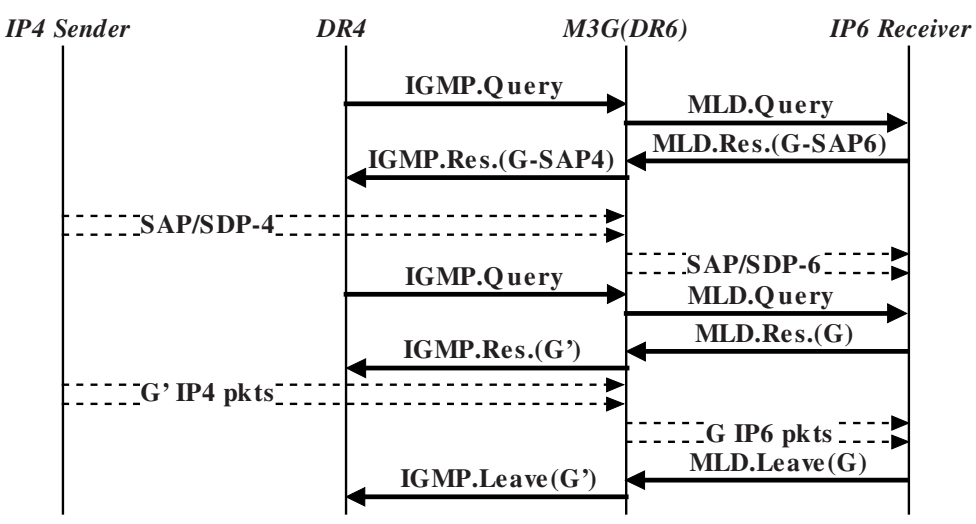

Fig. 4. Explain communication from one IPv4 multicast multimedia sender node to one or more IPv6 multicast receivers nodes based on SAP/SDP signalization.

\section{Global Functioning Example}

The communication between the sender and the receiver can be done in two steps:

Joining a multicast group. The translation of IGMP packets allows IPv6 receivers, implementing the MLD protocol, to join naturally multicast session. M3G, in that case, behaves as a designated router (DR6) for IPv6 receivers but in reality, it's the router behind (DR4) who is the designated router for IPv6 receivers.

Thus, IPv6 receivers will receive SAP/SDP signaling flows. The IPv6 receivers will be able to join in the same way another multimedia multicast session.

Leaving a multicast group. When leaving a multicast group, the IPv6 receivers' nodes send MLD Leave request. This request must not be handled at our gateway but it must be translated to IGMP Leave request. So, there is no state at our gateway about multicast receivers.

\section{Performance Analysis}

M3G's implementation is evaluated over a test platform using various network configurations. We emphasize the scalability and efficiency of our proposal using several MPEG-4 communication sessions. Each session is generated by a server attached to the wired IPv4 network, while the clients are located in the wireless IPv6 network behind an access point (802.11b) attached to the M3G (Fig. 2) entity.

According to this scenario, the server generates RTP sessions and their associated SAP/SDP signaling streams.

In our evaluation, we use an MPEG-4 with different spatial resolutions. This MPEG-4 video configuration, with a reduced complexity, is much suitable for mobile handled terminals. Also, we use different audio streams configuration ranging from MPEG-2 Audio layer 3 (mp3) to MPEG-4 Audio (aac), with different bit rates and sampling rates in order to get more information about the $\mathrm{M} 3 \mathrm{G}$ behavior. Based on a 
predictive coding, these media streams are very sensitive to the delayed packets since the packet with a too large latency will be discarded. In addition to these constraints, the delay variations (jitter) affect directly the perceived quality at client side.

All the components of our platform (i.e. servers, M3G gateway, and handled client) are running on a Linux (kernel 2.4.18) operating system. Both the gateway and the client are patched with USAGI pack for IPv6 support.

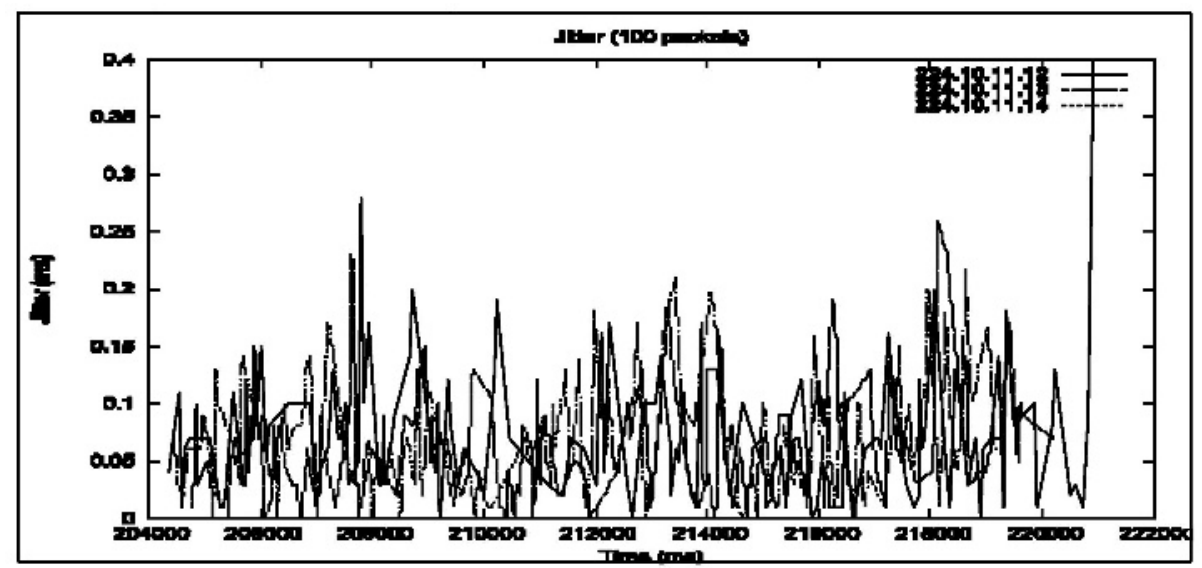

Fig. 5. Is a graphical representation of the jitter in the same environment as described above. We observe that the delay variation (jitter) for the processing of 100 packets remains under an acceptable threshold, the maximum being $0.38 \mathrm{~ms}$, and the average around $0.07 \mathrm{~ms}$.

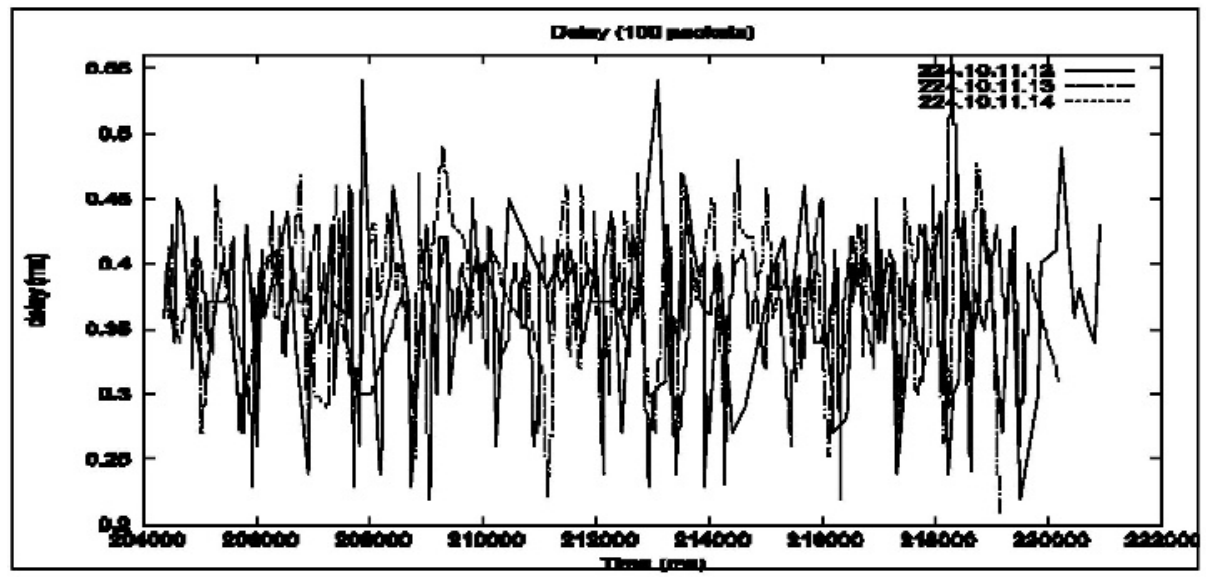

Fig. 6. Represents the delay induced by the treatment process of our gateway for RTP media packets. The Y-axes represents the delay in milliseconds for the processing of 100 packets and the $\mathrm{X}$-axes represents the time scale related to this communication session. Our gateway doesn't introduce such a delay variation, which could lead to a too important packet discarding rate. 
For further analyses and details about the performance evaluation of the M3G, we recommend to take a look to the internal report [19].

\section{Conclusion}

This article provides open issues on the smooth and efficient support of multicast multimedia services involving heterogeneous mobile IPv4 and IPv6 hosts. After analyzing related works on this broad area as well as existing but limited and partial solutions, we have proposed and evaluated an integrated Multicast Media Gateway for IPv4/IPv6 Multimedia Service Transition.

The overall M3G's architecture and associated functional modules have been described and implemented on a Linux-based system for features validation and performance evaluation. Transition issues regarding the user and the signaling control plans have been both addressed in this work.

A proposal on an interaction mechanism between IPv4 and IPv6 clouds for the automation of the access to multicast groups by wireless IPv6 hosts has been developed. Next, an implementation of Application Level Gateways (ALG) has been brought for SAP/SDP and RTSP in order to support both IP multimedia service types: (1) interactive unicast (e.g. VOD) and (2) non-interactive multicast (e.g. TV broadcasting) access modes.

Regarding to the mobility aspect of such services, new approaches and improvements have been added to the signaling plan through a better integration of existing multimedia session management, mobility and multicast signaling protocols. Consequently, transparent establishment and efficient control of hybrid multimedia multicast communications between mobile IPv4-only and IPv6-only clouds are possible while preserving legacy IPv4 networks and services during a smooth IPv6 transition.

\section{References}

1. Nordmark, E.: Stateless IP/ICMP Translation Algorithm (SIIT). RFC2765 (2000).

2. Handley, M., Perkins, C., Whelan, E.: Session Announcement Protocol (SAP). RFC2974 (2000)

3. Handley, M., Jacobson, V.: Session Description Protocol (SDP). RFC2327 (1998).

4. Olson, S., Camarillo, G., Roach, A. B.: Support for IPv6 in Session Description Protocol (SDP). RFC3266 (2002)

5. Tsirtsis, G., Srisuresh, P.: Network Address Translation - Protocol Translation (NAT-PT). RFC2766 (2000)

6. Tsuchiya, K., Higuchi, H., Sawada, S., Nozaki, S.: MTP: An IPv6/IPv4 Multicast Translator based on IGMP/MLD Proxying". draft-ietf-ngtrans-mtp-03.txt (2002)

7. Hallin, P., Satapati, S.: NAT-PT DNS ALG solutions. draft-hallin-natpt-dns-alg-solutions01 (2002)

8. Fenner, W.: Internet Group Management Protocol, Version 2 (IGMPv2). RFC2236 (1997)

9. Deering, S., Fenner, W., Haberman, B.: Multicast Listener Discovery (MLD). RFC2710 (1999)

10. Schulzrinne, H., Casner, S., Frederick, R., Jacobson, V.: A Transport Protocol for RealTime Applications. RFC1889 (1996) 
11. Bradner, S., Mankin, A.: The Recommendation for the IP Next Generation Protocol. RFC1752 (1995)

12. Deering, S., Hinden, R.: Internet Protocol, Version 6 (IPv6) - Specification. RFC1883 (1995)

13. King, S., Fax, R., Haskin, D., Ling, W., Fink, R., Meehan, T., Perkins, C. E.: The Case for IPv6. draft-iab-case-for-ipv6-06.txt (2000)

14. Gilligan, R., Nordmark, E.: Transition Mechanisms for IPv6 Hosts and Routers. RFC2893 (2000)

15. Johnson, D., Perkins, C., Arkko, J.: Mobility Support in IPv6. draft-ietf-mobileip-ipv621.txt (2003)

16. Perkins, C.: IP Mobility Support. RFC 2002, Mobile IP Networking Group (1996)

17. Harrison, T., Williamson, C., Mackrell, W., Bunt, R.: Mobile multicast (MOM) protocol: multicast support for mobile hosts. in Proc. Of ACM MOBICOM'97 (1997) 151-160.

18. Crawford, M.: Transmission of IPv6 Packets over Ethernet Networks. RFC2464 (1998)

19. Hadjadj Aoul, Y., Negru, D., Nafaa, A., Mehaoua, A.: M3G: Deploying Multimedia Services for Next Generation Networks. Technical Report PRiSM Lab., University of Versailles, France (2003). 